\title{
An E(s²)-Optimal Supersaturated Designs Constructed from Bulutoglu and Cheng Theorem
}

\author{
J. I. Mbegbu' ${ }^{1 *}$, C. O. Todo ${ }^{2}$ \\ ${ }^{1}$ Department of Mathematics, University of Benin, Benin City, Nigeria \\ ${ }^{2}$ Department of Statistics, School of Computing and IT, Delta State Polytechnic, Otefe-Oghara, Nigeria
}

\begin{abstract}
We constructed an $E\left(s^{2}\right)$-optimal supersaturated design (SSD) with an experimental run-size, $\mathrm{n}=20$ and number of factors, $m=57$ (multip le of 19). The construction is based on balanced incomplete block designs using a theorem proposed by Bulutoglu and Cheng. This is achieved by constructing the initial blocks of the balanced inco mplete block design using the theorem. Consequently, all other blocks are generated to constitute a balanced incomplete block design (BIBD),and the complete blocks that constitute the BIBD is transformed into the required supersaturated design( SSD).
\end{abstract}

Keywords $E\left(s^{2}\right)$-Optimal, Supersaturated, Design Matrix, Incomp lete Block Designs, Run-Size

\section{Introduction}

Consider a two-level experimental design with $n$ experimental runs and $\mathrm{m}$ factors. A two-level experimental design is called a supersaturated design(SSD) if $m>n-1$. According to[7], the analysis of supersaturated designs rely on the effect of sparsity principle in which the number of important factors is relatively small.

In[11], the $E\left(s^{2}\right)$ criterion is considered as the most popular criterion, and it measures the average correlation among the columns of the design matrix of an SSD. Suppose we have a design with $n$ runs and $m$ factors, and each of the factors has 2 levels, then $\frac{n}{2}$ of the entries in each column have values +1 , and others -1 respectively. Let $X$ denotes the $n \times m$ design matrix. Hence,

$$
E\left(s^{2}\right)=\frac{\sum_{i<j} s_{i j}^{2}}{\left(\begin{array}{l}
m \\
2
\end{array}\right)}
$$

where

$S_{i j}$ denotes the element in the ith row and jth column of $X^{T} X$.

For the supersaturated design with $\mathrm{n}$ runs and $\mathrm{m}$ factors,

* Corresponding author:

julian.mbegbu@yahoo.com (J. I. Mbegbu)

Published online at http://journal.sapub.org/ajms

Copyright (C) 2012 Scientific \& Academic Publishing. All Rights Reserved each column of the design matrix contains the same number of 1's and -1's. The number of possible factors, $m$ that could be accommodated satisfies the inequality

$$
n-1<m \leq\left(\begin{array}{l}
n-1 \\
\frac{n}{2}-1
\end{array}\right)
$$

[8] showed that when $n$ is even:

$$
E\left(s^{2}\right) \geq \frac{m\left(n^{2}+n-1\right)-n^{3}}{n(m-1)}
$$

According to[7], the lower bound (1.3) is attainable when $\mathrm{n}$ is a multiple of 4 and $\mathrm{m}$ is a multiple of $n-1$, or $n=2$ ( $\bmod 4)$ or $\mathrm{m}$ is an even multiple of $n-1$.

Moreover,[6] and[8] independently derived the lower bound:

$$
E\left(s^{2}\right) \geq \frac{n^{2}\left(m^{2}-n+1\right)}{(m-1)(n-1)}
$$

for any supersaturated design with $\mathrm{m}$ factors and $\mathrm{n}$ runs.

[1] proposed the $E\left(s^{2}\right)$ criterion for constructing two-level supersaturated designs, and[8] based the construction of supersaturated designs on combining q Hadamard matrices. This means that a simple construction of an SSD is to piece together q balanced incomplete balanced block designs( BIBD's) $\left(n-1, q(n-1), \frac{n}{2}-1\right)$. Thus, the resulting SSD will have distinct blocks.[9] presented a method of using difference family to construct BIBD's $\left(n-1, q(n-1), \frac{n}{2}-1\right)$ with distinct blocks, and from such a BIBD one could easily construct an $E\left(s^{2}\right)$-optimal SSD. 
[10] introduced the construction of SSD's that satisfy specified lower bounds of $E\left(s^{2}\right)$,and[12] highlighted ways of constructing optimal mix-level supersaturated designs. In[4], a column wise-pairwise algorithm for constructing SSD's is discussed.

In this study, we shall construct an $E\left(s^{2}\right)$-optimal supersaturated design (SSD) with an even run-size, $n$, and number of factors, a multiple of $n-1$.

\section{Materials and Method}

Theorem[2]: Suppose $n-1$ is an odd prime power, $q$ an even divisor of $n-2$ with $q \neq n-2, x$ a primitive element of $G F(n-1)$, and $T$ a subset of $Z_{q}$ of size $\frac{q}{2}$. Let $\ell$ be the smallest positive integersuch that $T+e=T$. Then, the $e(n-1) \quad$ sets: $\left\{S_{r, a}: r=0, \mathrm{~K}, e-1, a \in G F(n-1)\right\} \quad, \quad$ where $S_{r, a}=\left\{x^{j q+i}+a: 0 \leq j \leq \frac{(n-2)}{q}-1, i \in T+r\right\}$ are distinct and constitute a BIBD $\left(n-1, e(n-1), \frac{n}{2}-1\right)$. Furthermore, if $\frac{(n-2)}{q}$ is odd, and $U$ a subset of $\{0, \mathrm{~L}, e-1\}$ of size $\frac{e}{2}$ such that $U^{*}=U+\left(\frac{q}{2}\right)$, where $U^{*}$ is the complement of $U$ in $\{0, \mathrm{~K}, e-1\}$ and the addition is reduced modulo $q$, then the $\frac{e(n-1)}{q}$ set : $\left\{\mathrm{S}_{\mathrm{r}, \mathrm{a}}: r \in U, a \in G F(n-1)\right\}$ constitutes a BIBD with distinct blocks.

The theorem satisfies the lower bound (1.4), and shall be used in the construction of the supersaturated design (SSD). The construction of the SSD will be based on BIBD, which is more general and flexible[6].According to[2], given a Hadamard matrix of order $\mathrm{n}$, there exists a BIBD $\left(n-1, q(n-1), \frac{n}{2}-1\right)$ for every $q>0$.

The following definitions shall be used in this work.

Definition 1: Let $G F(s)$ denotes a finite field with $s$ elements. An element in a group within a finite field from which other elements are obtained is called a generator of the group. The generator of a group is called a primitive element of the field.
Definition 2: Let $Z_{q}=\{0,1,2, \mathrm{~L}, q-1\}, q>0$.

Multiplication and addition in $Z_{q}$ are in reduced modulo q. Now, for each subset $T=\left\{a_{1}, a_{2}, \mathrm{~K}, a_{t}\right\}$ of $z_{q}$, and if $b \in z_{q}$, then $T+b=\left\{a_{1}+b, a_{2}+b, \mathrm{~K}, a_{t}+b\right\}$.

Definition 3: According to[5], an incomp lete blockdesign $D(v, b, k, r)$ is called a balanced incomplete block design (BIBD) if

(i) each block contains $\mathrm{k}$ different treatments

(ii) each treatment appears in $r$ blocks, that is, each treatment is rep licated $r$ times.

(iii) every pair of treatments appears within blocks exactly $\lambda$ times.

For a BIBD, according to[3]

(a) $b \geq v$ or $k \geq r$

(b) $b k=v r$

(c) : $r(k-1)=\lambda(v-1) ; r>\lambda$

Definition 4: We define a BIBD by its incidence matrix $N=\left[n_{i j}\right]$, where $n_{i j}=\left\{\begin{array}{l}1, \text { if the ith treatment appears in the jth block } \\ 0, \text { otherwise }\end{array}\right.$ Construction of an $E\left(s^{2}\right)$-Optimal Supersaturated

\section{Design:}

Let the primitive element $x=2$. Then, we shall construct a $\operatorname{BIBD}(19,57,9)$ in the following way:

Let the value of integer $q$ in the theorem be 6 . $T=U=\{0,1,2\}$ since $T$ is a subset of $Z_{6}$ of size 3 . There are many of such subsets of sizes 3 within $Z_{6}=\{0,1,2,3,4,5\}$. The number of subsets of sizes 3 in $Z_{6}$ is ${ }^{6} C_{3}=20$. The 20 subsets of $Z_{6}$ each of size 3 are: $\{0,1,2\},\{0,1,3\},\{0,1,4\},\{0,1,5\},\{0,2,3\},\{0,2,4\}$, $\{0,2,5\},\{0,3,4\},\{0,3,5\},\{0,4,5\},\{1,2,3\},\{1,2,4\},\{1$, $2,5\},\{1,3,4\},\{1,3,5\},\{1,4,5\},\{2,3,4\},\{2,3,5\},\{2,4$, $5\},\{3,4,5\}$.Hence, $\mathrm{T}+\mathrm{e}=\mathrm{T}+3=\mathrm{T}$ for all these subsets except $\{0,1,2\}$. For the subset $\{0,1,2\}, e=3$.

We now obtain the initial blocks of the BIBD as follows: For $q=6$, by the Theorem :

$$
0 \leq j \leq \frac{(n-2)}{q}-1
$$

which reduces to $0 \leq j \leq 2$, for $n=20$. Since, $e=3$ and $i \in T+r,(r=0,1,2)$, then $a \in G F(n-1)=G F(19)=G F(0,1,2,3,4,5,6,7,8$, $9,10,11,12,13,14,15,16,17,18)$. 
Considering the $S_{r, a}=\left\{x^{j q+i}+a: 0 \leq j \leq \frac{n-2}{q}-1, i \in T+r\right\} \quad$, and evaluating $x^{j q+i}$, for $j=0,1,2 ; i=0,1,2 ; q=6$; $e=3 ; r=0,1,2$, we obtain Table 1 .

Table 1. Blocks

\begin{tabular}{|c|c|c|}
\hline$x^{j q+i}$ & $x^{j q+i}$ & $x^{j q+i}$ \\
\hline $\begin{array}{c}\text { when } \\
r=0, i \in(0,1,2)\end{array}$ & $\begin{array}{c}\text { when } \\
j=1, i \in(1,2,3)\end{array}$ & when \\
$j=0$ & $j=0$ & $r=2, i \in(2,3,4)$ \\
$x^{0}, x^{1}, x^{2}$ & $x^{1}, x^{2}, x^{3}$ & $j=0$ \\
$j=1$ & $j=1$ & $x^{2}, x^{3}, x^{4}$ \\
$j=1$ \\
$x^{6}, x^{7}, x^{8}$ & $x^{7}, x^{8}, x^{9}$ & $x^{8}, x^{9}, x^{10}$ \\
$j=2$ \\
$j=2$ & $j=2$ & $x^{14}, x^{15}, x^{16}$ \\
$x^{12}, x^{13}, x^{14}$ & $x^{13}, x^{14}, x^{15}$ & \\
\hline
\end{tabular}

\section{Results and Discussion}

With the primitive element $x=2$, Table 1 contains three initial blocks namely, $\left\{2^{0}, 2^{1}, 2^{2}, 2^{6}, 2^{7}, 2^{8}, 2^{12}, 2^{13}, 2^{14}\right\},\left\{2^{1}\right.$, $\left.2^{2}, 2^{3}, 2^{7}, 2^{8}, 2^{9}, 2^{13}, 2^{14}, 2^{15}\right\}$ and $\left\{2^{2}, 2^{3}, 2^{4}, 2^{8}, 2^{9}, 2^{10}, 2^{14}, 2^{15}\right.$, $\left.2^{16}\right\}$. As multiplication is in reduced modulo $(n-1)$, i.e., reduced modulo 19 , the first initial block contains: $\left\{2^{0}, 2^{1}\right.$, $\left.2^{2}, 2^{6}, 2^{7}, 2^{8}, 2^{12}, 2^{13}, 2^{14}\right\}$, where $2^{0}=1,2^{1}=2$, $2^{2}=4$,

$2^{6}=64=7(\bmod 19), 2^{7}=128=14(\bmod 19)$,

$2^{8}=256=9(\bmod 19), 2^{12}=4096=11(\bmod 19)$,

$2^{13}=8192=3(\bmod 19), 2^{14}=16384=6(\bmod 19)$.

Therefore, the first in itial b lock (FIB) is $\{1,2,4,7,14,9$, $11,6\}$

From Table 1, the second initial block (SIB) contains: $\left\{2^{1}\right.$, $\left.2^{2}, 2^{3}, 2^{7}, 2^{8}, 2^{9}, 2^{13}, 2^{14}, 2^{15}\right\}$, where $2^{1}=2,2^{2}=4$, $2^{3}=8$,

$2^{7}=128=14(\bmod 19), 2^{8}=256=9(\bmod 19)$,

$2^{9}=512=18(\bmod 19), 2^{13}=8192=3(\bmod 19)$,

$2^{14}=16384=6(\bmod 19), 2^{15}=32768=12(\bmod 19)$.

Therefore, the second initial b lock is $\{2,4,8,14,9,18,3$, $6,12\}$

When the above procedure is repeated for the third initial block, we have the third initial block (TIB) as $\{4,8,16,9$, $18,17,6,12,5\}$.

We note that in the three initial blocks, the numbers indicate the treatments that occur in each block. In th is case, the three initial blocks are shown in Table 2 .
Table 2. The Three Initial Blocks

\begin{tabular}{|c|c|c|c|}
\hline & & Blocks & \\
\hline Treatments & FIB & SIB & TIB \\
1 & 1 & 0 & 0 \\
2 & 1 & 1 & 0 \\
3 & 1 & 1 & 0 \\
4 & 1 & 1 & 1 \\
5 & 0 & 0 & 1 \\
6 & 1 & 1 & 1 \\
7 & 1 & 0 & 0 \\
8 & 0 & 1 & 1 \\
9 & 1 & 1 & 1 \\
10 & 0 & 0 & 0 \\
11 & 1 & 0 & 0 \\
12 & 0 & 1 & 1 \\
13 & 0 & 0 & 0 \\
14 & 1 & 1 & 0 \\
15 & 0 & 0 & 0 \\
16 & 0 & 0 & 1 \\
17 & 0 & 0 & 1 \\
18 & 0 & 1 & 1 \\
19 & 0 & 0 & 0 \\
\hline
\end{tabular}

Now, consider $x^{j q+i}+a$, where $a \in G F(19)$; that is, adding the integers $0,1, \ldots, 18(\bmod 19)$ to all the elements in the three initial blocks produces a BIBD with 57 distinct blocks each of size 9 and 19 blocks. From the First Initial Blocks:

$\mathrm{FIB}+0=\{1,2,4,7,14,9,11,3,6\}, \mathrm{FIB}+1=\{2,3,5,8$, $15,10,12,4,7\}$,

FIB $+2=\{3,4,6,9,16,11,13,5,8\}$, FIB $+3=\{4,5,7$, $10,17,12,14,6,9\}$,

$\mathrm{FIB}+4=\{5,6,8,11,18,13,15,7,10\}, \mathrm{FIB}+5=\{6,7,9$, $12,19,14,16,8,11\}$,

$\mathrm{FIB}+6=\{7,8,10,13,1,15,17,9,12\}, \mathrm{FIB}+7=\{8,9,11$, $14,2,16,18,10,13\}$,

FIB $+8=\{9,10,12,15,3,17,19,11,14\}, F I B+9=\{10$, $11,13,16,4,18,1,12,15\}$,

$\mathrm{FIB}+10=\{11,12,14,17,5,19,2,13,16\}, \mathrm{FIB}+11=$ $\{12,13,15,18,6,1,3,14,17\}$,

$\mathrm{FIB}+12=\{13,14,16,19,7,2,4,15,18\}, \mathrm{FIB}+13=\{14$, $15,17,1,8,3,5,16,19\}$,

$\mathrm{FIB}+14=\{15,16,18,2,9,4,6,17,1\}, \mathrm{FIB}+15=\{16$, $17,19,3,10,5,7,18,2\}$,

$\mathrm{FIB}+16=\{17,18,1,4,11,6,8,19,3\}, \mathrm{FIB}+17=\{18$, $19,2,5,12,7,9,1,4\}$,

FIB $+18=\{19,1,3,6,13,8,10,2,5\}$.

The above procedure is repeated on the Second Initial Block and the Third Initial Block respectively, altogether gives the BIBD (19, 57, 9). The First 19 blocks of the BIBD $(19,57,9)$ obtained from the First Initial Block are shown in Table 3 below .

Adopting the $\operatorname{BIBD}(19,57,9)$, we obtain a $19 \times 57$ treatment-block incidence matrix for $\operatorname{BIBD}(19,57,9)$. Using $\operatorname{SSD}(n, m)=\operatorname{SSD}(n, e(n-1))$, we obtain an $\operatorname{SSD}(20,57)$ from the $\operatorname{BIBD}(19,57,9)$ by converting all 0 's in the incidence matrix of the $\operatorname{BIBD}(19,57,9)$ to -1 's and adding a first row of all 1's to the treatment-block incidence matrix. This gives 
an $E\left(s^{2}\right)$-optimal 20-run SSD for 57 factors shown in Table 4 below.

Table 4 shows the complete 57 factors of the $\operatorname{SSD}(20,57)$ constructed from the $\operatorname{BIBD}(19,57,9)$. We observe that all the two level factors occur in the higher level for the first run (run 1) of the design.

Table 4 shows the complete 57 factors of the $\operatorname{SSD}(20,57)$ constructed from the $\operatorname{BIBD}(19,57,9)$. We observe that all the two level factors occur in the higher level for the first run (run 1) of the design.

Table 3. First 19 blocks of the $\operatorname{BIBD}(19,57,9)$ obtained from the first initial block

\begin{tabular}{|c|c|c|c|c|c|c|c|c|c|c|c|c|c|c|c|c|c|c|c|}
\hline \multicolumn{20}{|c|}{ Blocks } \\
\hline \multirow[t]{3}{*}{$\begin{array}{l}\text { Treat- } \\
\text { ments }\end{array}$} & 1 & 2 & 3 & 4 & 5 & 6 & 7 & 8 & 9 & 10 & 11 & 12 & 13 & 14 & 15 & 16 & 17 & 18 & 19 \\
\hline & + & + & + & + & + & + & + & + & + & + & + & + & + & + & + & + & + & + & + \\
\hline & 0 & 1 & 2 & 3 & 4 & 5 & 6 & 7 & 8 & 9 & 10 & 11 & 12 & 13 & 14 & 15 & 16 & 17 & 18 \\
\hline 1 & 1 & 0 & 0 & 0 & 0 & 0 & 1 & 0 & 0 & 1 & 0 & 1 & 0 & 1 & 1 & 0 & 1 & 1 & 1 \\
\hline 2 & 1 & 1 & 0 & 0 & 0 & 0 & 0 & 1 & 0 & 0 & 1 & 0 & 1 & 0 & 1 & 1 & 0 & 1 & 1 \\
\hline 3 & 1 & 1 & 1 & 0 & 0 & 0 & 0 & 0 & 1 & 0 & 0 & 1 & 0 & 1 & 0 & 1 & 1 & 1 & 1 \\
\hline 4 & 1 & 1 & 1 & 1 & 0 & 0 & 0 & 0 & 0 & 1 & 0 & 0 & 1 & 0 & 1 & 0 & 1 & 1 & 0 \\
\hline 5 & 0 & 1 & 1 & 1 & 1 & 0 & 0 & 0 & 0 & 0 & 1 & 0 & 0 & 1 & 0 & 1 & 0 & 1 & 1 \\
\hline 6 & 1 & 0 & 1 & 1 & 1 & 1 & 0 & 0 & 0 & 0 & 0 & 1 & 0 & 0 & 1 & 0 & 1 & 0 & 1 \\
\hline 7 & 1 & 1 & 0 & 1 & 1 & 1 & 1 & 0 & 0 & 0 & 0 & 0 & 1 & 0 & 0 & 1 & 0 & 0 & 0 \\
\hline 8 & 0 & 1 & 1 & 0 & 1 & 1 & 1 & 1 & 0 & 0 & 0 & 0 & 0 & 1 & 0 & 0 & 1 & 0 & 1 \\
\hline 9 & 1 & 0 & 1 & 1 & 0 & 1 & 1 & 1 & 1 & 0 & 0 & 0 & 0 & 0 & 1 & 0 & 0 & 1 & 0 \\
\hline 10 & 0 & 1 & 0 & 1 & 1 & 0 & 1 & 1 & 1 & 1 & 0 & 0 & 0 & 0 & 0 & 1 & 0 & 0 & 1 \\
\hline 11 & 1 & 0 & 1 & 0 & 1 & 1 & 0 & 1 & 1 & 1 & 1 & 0 & 0 & 0 & 0 & 0 & 0 & 0 & 0 \\
\hline 12 & 0 & 1 & 0 & 1 & 0 & 1 & 0 & 0 & 1 & 1 & 1 & 1 & 0 & 0 & 0 & 0 & 0 & 1 & 0 \\
\hline 13 & 0 & 0 & 1 & 0 & 1 & 0 & 1 & 1 & 0 & 1 & 1 & 1 & 1 & 0 & 0 & 0 & 0 & 0 & 1 \\
\hline 14 & 1 & 0 & 0 & 1 & 0 & 1 & 0 & 1 & 1 & 0 & 1 & 1 & 1 & 0 & 0 & 0 & 0 & 0 & 0 \\
\hline 15 & 0 & 1 & 0 & 0 & 1 & 0 & 1 & 0 & 1 & 1 & 0 & 1 & 1 & 1 & 1 & 0 & 0 & 0 & 0 \\
\hline 16 & 0 & 0 & 1 & 0 & 0 & 1 & 0 & 1 & 0 & 1 & 1 & 0 & 1 & 1 & 1 & 1 & 0 & 0 & 0 \\
\hline 17 & 0 & 0 & 0 & 1 & 0 & 0 & 1 & 0 & 1 & 0 & 1 & 1 & 0 & 1 & 1 & 1 & 1 & 0 & 0 \\
\hline 18 & 0 & 0 & 0 & 0 & 1 & 0 & 0 & 1 & 0 & 1 & 0 & 1 & 1 & 0 & 1 & 1 & 1 & 1 & 0 \\
\hline 19 & 0 & 0 & 0 & 0 & 0 & 1 & 0 & 0 & 1 & 0 & 1 & 0 & 1 & 1 & 0 & 1 & 1 & 1 & 1 \\
\hline
\end{tabular}

Table 4. The complete 57 factors of the $\operatorname{SSD}(20,57)$ constructed from the $\operatorname{BIBD}(19,57,9)$

\begin{tabular}{|c|c|c|c|c|c|c|c|c|c|c|c|c|c|c|c|c|c|c|c|}
\hline & \multicolumn{19}{|c|}{ Factors } \\
\hline Runs & 1 & 2 & 3 & 4 & 5 & 6 & 7 & 8 & 9 & 10 & 11 & 12 & 13 & 14 & 15 & 16 & 17 & 18 & 19 \\
\hline 1 & 1 & 1 & 1 & 1 & 1 & 1 & 1 & 1 & 1 & 1 & 1 & 1 & 1 & 1 & 1 & 1 & 1 & 1 & 1 \\
\hline 2 & 1 & -1 & -1 & -1 & -1 & -1 & 1 & -1 & -1 & 1 & -1 & 1 & -1 & 1 & 1 & -1 & 1 & 1 & 1 \\
\hline 3 & 1 & 1 & -1 & -1 & -1 & -1 & -1 & 1 & -1 & -1 & 1 & -1 & 1 & -1 & 1 & 1 & -1 & 1 & -1 \\
\hline 4 & 1 & 1 & 1 & -1 & -1 & -1 & -1 & -1 & 1 & -1 & -1 & 1 & -1 & 1 & -1 & 1 & 1 & 1 & 1 \\
\hline 5 & 1 & 1 & 1 & -1 & -1 & -1 & -1 & -1 & -1 & 1 & -1 & -1 & 1 & -1 & 1 & -1 & 1 & 1 & -1 \\
\hline 6 & -1 & 1 & 1 & 1 & 1 & -1 & -1 & -1 & -1 & -1 & 1 & -1 & $\begin{array}{l}-1 \\
\end{array}$ & 1 & -1 & -1 & -1 & 1 & 1 \\
\hline 7 & 1 & -1 & 1 & 1 & 1 & 1 & -1 & -1 & -1 & -1 & -1 & 1 & -1 & -1 & 1 & -1 & 1 & -1 & 1 \\
\hline 8 & 1 & 1 & -1 & 1 & 1 & 1 & 1 & -1 & -1 & -1 & -1 & -1 & 1 & -1 & -1 & 1 & -1 & 1 & -1 \\
\hline 9 & -1 & 1 & 1 & 1 & -1 & 1 & 1 & 1 & -1 & -1 & -1 & -1 & -1 & 1 & -1 & -1 & 1 & -1 & 1 \\
\hline 10 & 1 & -1 & 1 & -1 & -1 & -1 & 1 & 1 & 1 & -1 & -1 & -1 & -1 & -1 & 1 & -1 & -1 & 1 & -1 \\
\hline 11 & -1 & 1 & -1 & 1 & 1 & -1 & 1 & 1 & 1 & 1 & -1 & -1 & -1 & -1 & -1 & 1 & -1 & -1 & 1 \\
\hline 12 & 1 & -1 & 1 & 1 & 1 & 1 & -1 & 1 & 1 & 1 & 1 & -1 & -1 & -1 & -1 & -1 & 1 & -1 & -1 \\
\hline 13 & -1 & 1 & -1 & -1 & -1 & 1 & 1 & -1 & 1 & 1 & 1 & 1 & $\begin{array}{l}-1 \\
\end{array}$ & $\begin{array}{l}-1 \\
\end{array}$ & -1 & -1 & -1 & 1 & -1 \\
\hline 14 & -1 & -1 & 1 & 1 & 1 & $\begin{array}{l}-1 \\
\end{array}$ & 1 & 1 & -1 & 1 & 1 & 1 & 1 & $\begin{array}{l}-1 \\
\end{array}$ & $\begin{array}{l}-1 \\
\end{array}$ & $\begin{array}{l}-1 \\
\end{array}$ & -1 & -1 & 1 \\
\hline 15 & 1 & -1 & -1 & 1 & -1 & 1 & -1 & 1 & 1 & -1 & 1 & 1 & 1 & 1 & -1 & -1 & -1 & $\begin{array}{l}-1 \\
\end{array}$ & -1 \\
\hline 16 & -1 & 1 & -1 & -1 & 1 & -1 & 1 & -1 & 1 & 1 & -1 & 1 & 1 & 1 & 1 & -1 & -1 & -1 & -1 \\
\hline 17 & -1 & -1 & 1 & -1 & -1 & 1 & -1 & 1 & -1 & 1 & 1 & -1 & 1 & 1 & 1 & 1 & -1 & -1 & -1 \\
\hline 18 & -1 & -1 & -1 & 1 & -1 & -1 & 1 & -1 & 1 & -1 & 1 & 1 & -1 & 1 & 1 & 1 & 1 & -1 & -1 \\
\hline 19 & -1 & -1 & -1 & -1 & 1 & -1 & -1 & 1 & -1 & 1 & -1 & 1 & 1 & -1 & 1 & 1 & 1 & 1 & -1 \\
\hline 20 & -1 & -1 & -1 & -1 & -1 & 1 & -1 & -1 & 1 & -1 & 1 & 1 & 1 & 1 & -1 & 1 & 1 & 1 & 1 \\
\hline
\end{tabular}


Table 4. Cont'd

\begin{tabular}{|c|c|c|c|c|c|c|c|c|c|c|c|c|c|c|c|c|c|c|c|c|}
\hline & \multicolumn{20}{|c|}{ Factors } \\
\hline Runs & 20 & 21 & 22 & 23 & 24 & 25 & 26 & 27 & 28 & 29 & 30 & 31 & 32 & 33 & 34 & 35 & 36 & 37 & 38 & 39 \\
\hline 1 & 1 & 1 & 1 & 1 & 1 & 1 & 1 & 1 & 1 & 1 & 1 & 1 & 1 & 1 & 1 & 1 & 1 & 1 & 1 & 1 \\
\hline 2 & -1 & -1 & 1 & -1 & -1 & -1 & 1 & -1 & -1 & -1 & -1 & 1 & 1 & -1 & 1 & -1 & 1 & 1 & 1 & -1 \\
\hline 3 & -1 & -1 & -1 & 1 & -1 & -1 & -1 & 1 & -1 & 1 & -1 & -1 & 1 & 1 & -1 & 1 & 1 & 1 & 1 & -1 \\
\hline 4 & 1 & -1 & -1 & -1 & 1 & -1 & -1 & -1 & 1 & -1 & 1 & -1 & -1 & 1 & 1 & -1 & 1 & 1 & 1 & -1 \\
\hline 5 & 1 & 1 & 1 & -1 & -1 & 1 & -1 & -1 & -1 & 1 & -1 & 1 & -1 & -1 & 1 & 1 & -1 & 1 & 1 & 1 \\
\hline 6 & -1 & 1 & 1 & 1 & -1 & -1 & 1 & -1 & -1 & -1 & 1 & -1 & 1 & -1 & -1 & 1 & 1 & -1 & 1 & 1 \\
\hline 7 & 1 & -1 & 1 & 1 & 1 & -1 & -1 & 1 & -1 & -1 & -1 & 1 & -1 & 1 & -1 & -1 & 1 & 1 & -1 & 1 \\
\hline 8 & -1 & -1 & -1 & 1 & 1 & 1 & -1 & -1 & 1 & -1 & -1 & -1 & 1 & -1 & 1 & -1 & -1 & 1 & 1 & -1 \\
\hline 9 & 1 & -1 & 1 & -1 & 1 & 1 & 1 & -1 & -1 & 1 & -1 & -1 & -1 & 1 & -1 & 1 & -1 & -1 & 1 & -1 \\
\hline 10 & 1 & 1 & -1 & 1 & -1 & 1 & 1 & 1 & -1 & -1 & 1 & -1 & -1 & -1 & 1 & -1 & 1 & -1 & -1 & 1 \\
\hline 11 & -1 & 1 & 1 & -1 & 1 & -1 & 1 & 1 & 1 & -1 & -1 & 1 & -1 & -1 & -1 & 1 & -1 & 1 & -1 & -1 \\
\hline 12 & -1 & -1 & 1 & 1 & -1 & 1 & -1 & 1 & 1 & 1 & -1 & -1 & 1 & -1 & -1 & -1 & 1 & -1 & 1 & -1 \\
\hline 13 & 1 & -1 & -1 & 1 & 1 & -1 & 1 & -1 & 1 & 1 & 1 & -1 & -1 & 1 & -1 & -1 & -1 & 1 & -1 & -1 \\
\hline 14 & -1 & 1 & -1 & -1 & 1 & 1 & -1 & 1 & -1 & 1 & 1 & 1 & -1 & -1 & 1 & -1 & -1 & -1 & 1 & -1 \\
\hline 15 & 1 & 1 & 1 & -1 & -1 & 1 & 1 & -1 & 1 & -1 & -1 & -1 & -1 & 1 & -1 & 1 & -1 & -1 & -1 & -1 \\
\hline 16 & -1 & 1 & -1 & 1 & -1 & -1 & 1 & 1 & -1 & 1 & -1 & 1 & 1 & 1 & -1 & -1 & 1 & -1 & -1 & -1 \\
\hline 17 & -1 & -1 & 1 & -1 & 1 & -1 & -1 & 1 & 1 & -1 & 1 & -1 & 1 & 1 & 1 & -1 & -1 & 1 & $\begin{array}{l}-1 \\
\end{array}$ & 1 \\
\hline 18 & -1 & -1 & 1 & 1 & -1 & 1 & -1 & -1 & 1 & 1 & -1 & 1 & -1 & 1 & 1 & 1 & -1 & -1 & 1 & 1 \\
\hline 19 & 1 & -1 & 1 & -1 & 1 & -1 & -1 & 1 & 1 & -1 & 1 & -1 & 1 & -1 & 1 & 1 & 1 & -1 & -1 & 1 \\
\hline 20 & -1 & 1 & -1 & -1 & -1 & 1 & -1 & 1 & -1 & -1 & 1 & 1 & -1 & 1 & -1 & 1 & 1 & 1 & -1 & -1 \\
\hline
\end{tabular}

Table 4. Cont' $d$

\begin{tabular}{|c|c|c|c|c|c|c|c|c|c|c|c|c|c|c|c|c|c|c|}
\hline & \multicolumn{18}{|c|}{ Factors } \\
\hline Runs & 40 & 41 & 42 & 43 & 44 & 45 & 46 & 47 & 48 & 49 & 50 & 51 & 52 & 53 & 54 & 55 & 56 & 57 \\
\hline 1 & 1 & 1 & 1 & 1 & 1 & 1 & 1 & 1 & 1 & 1 & 1 & 1 & 1 & 1 & 1 & 1 & 1 & 1 \\
\hline 2 & 1 & 1 & 1 & 1 & -1 & -1 & -1 & 1 & -1 & -1 & 1 & 1 & -1 & 1 & 1 & 1 & -1 & -1 \\
\hline 3 & -1 & -1 & 1 & 1 & 1 & -1 & -1 & -1 & 1 & -1 & 1 & 1 & 1 & -1 & 1 & 1 & 1 & -1 \\
\hline 4 & -1 & -1 & -1 & 1 & 1 & 1 & -1 & -1 & 1 & 1 & 1 & -1 & 1 & 1 & -1 & 1 & 1 & 1 \\
\hline 5 & -1 & -1 & -1 & -1 & 1 & 1 & 1 & -1 & -1 & -1 & 1 & -1 & -1 & 1 & 1 & -1 & 1 & 1 \\
\hline 6 & 1 & -1 & -1 & -1 & -1 & 1 & 1 & 1 & -1 & -1 & -1 & 1 & -1 & -1 & 1 & 1 & -1 & 1 \\
\hline 7 & 1 & 1 & -1 & -1 & -1 & -1 & 1 & 1 & 1 & -1 & -1 & -1 & 1 & -1 & -1 & 1 & 1 & -1 \\
\hline 8 & 1 & 1 & 1 & -1 & -1 & -1 & -1 & 1 & 1 & 1 & -1 & -1 & -1 & 1 & -1 & -1 & 1 & 1 \\
\hline 9 & -1 & 1 & 1 & 1 & -1 & -1 & -1 & -1 & 1 & 1 & 1 & -1 & -1 & -1 & 1 & -1 & -1 & 1 \\
\hline 10 & 1 & -1 & 1 & 1 & 1 & -1 & -1 & -1 & -1 & 1 & 1 & 1 & -1 & -1 & -1 & 1 & -1 & -1 \\
\hline 11 & 1 & 1 & -1 & 1 & 1 & 1 & -1 & -1 & -1 & -1 & 1 & 1 & 1 & -1 & -1 & -1 & 1 & -1 \\
\hline 12 & -1 & 1 & 1 & -1 & 1 & 1 & 1 & -1 & -1 & -1 & -1 & 1 & 1 & 1 & -1 & -1 & -1 & 1 \\
\hline 13 & -1 & -1 & 1 & 1 & -1 & 1 & 1 & 1 & -1 & -1 & -1 & -1 & 1 & 1 & 1 & -1 & -1 & -1 \\
\hline 14 & 1 & -1 & -1 & 1 & 1 & -1 & 1 & 1 & 1 & -1 & -1 & -1 & -1 & 1 & 1 & 1 & -1 & -1 \\
\hline 15 & -1 & 1 & -1 & -1 & 1 & 1 & -1 & 1 & 1 & 1 & -1 & -1 & -1 & -1 & 1 & 1 & 1 & -1 \\
\hline 16 & -1 & -1 & 1 & -1 & -1 & 1 & 1 & -1 & 1 & 1 & -1 & -1 & -1 & -1 & -1 & 1 & 1 & 1 \\
\hline 17 & -1 & -1 & -1 & 1 & -1 & -1 & 1 & 1 & -1 & 1 & 1 & 1 & -1 & -1 & -1 & -1 & 1 & 1 \\
\hline 18 & 1 & -1 & -1 & -1 & 1 & -1 & -1 & 1 & 1 & -1 & 1 & 1 & 1 & -1 & -1 & -1 & -1 & 1 \\
\hline 19 & 1 & 1 & -1 & -1 & -1 & 1 & -1 & -1 & 1 & 1 & -1 & 1 & 1 & 1 & -1 & -1 & -1 & -1 \\
\hline 20 & 1 & 1 & 1 & -1 & -1 & -1 & 1 & -1 & -1 & 1 & 1 & -1 & 1 & 1 & 1 & -1 & -1 & -1 \\
\hline
\end{tabular}

\section{Conclusions}

Using the primitive element of the field, GF(s) as defined in the work of[2], we constructed a supersaturated design, $\operatorname{SSD}(20,57)$ with 57 factors and 20 experimental runs. We determine the $E\left(s^{2}\right)$-value for the $\operatorname{SSD}(20,57)$ as 40.31 which satisfies the $E\left(s^{2}\right)$ lower bound proposed by[8]. This guarantees the validity of design, $\operatorname{SSD}(20,57)$. In the literature, a number of $\mathrm{E}\left(\mathrm{s}^{2}\right)$-optimal supersaturated design construction techniques have been utilised, but oftentimes, most are very rigorous, not adaptable and flexible. In this respect, constructing an $\mathrm{E}\left(\mathrm{s}^{2}\right)$-optimal supersaturated design from balanced incomplete block design excels. All the details of this construction technique have been successfully demonstrated in this paper. Obviously, this technique would 
be of great aid in the search for more $E\left(s^{2}\right)$-optimal supersaturated designs for factors screening in the preliminary stage of industrial or scientific experiments.

\section{REFERENCES}

[1] Booth, K. H. and Cox, D. R. (1962), "Some Systematic Supersaturated Designs”, Technometrics, Vol. 4, 489-495.

[2] Bulutoglu, D. A. and Cheng, C. (2004), “Construction of $E\left(s^{2}\right)$-optimal Supersaturated Designs”, The Annals of Statistics, Vol.32, No.4, August ,2004.

[3] Fisher, R. A. (1940), “An Examination of Different Possible Solutions of a Problem in Incomplete Blocks”, Annals of Eugenics, Vol. 10. pp. 52-57.

[4] Li, W. W. and Wu, C. F. J, (1997), "Columnwise-Pairwise Algorithm with Applications to the Construction of Supersaturated Designs”, Technometrics, Vol. 39: pp. 171-179 MR 1452345.

[5] Mbegbu, J. I. (2007). “Optimal Balanced Incomplete Block Designs with Cost Constraints”, A PhD Thesis, Rivers State University of Science and Technology, Port-Harcourt, Nigeria.
[6] Nguyen, N. K. (1996). “An Algorithmic Approach to Constructing Supersaturated Designs”, Technometrics, Vol. 38 pp. 67-73.

[7] Nguyen, N. K. and Cheng-Shul (2008), “New E(s2)-Optimal Supersaturated Designs Constructed from Incomplete Block Designs”, Technometrics, February 2008, Vol. 50, No. 1.

[8] Tang, B. X. and Wu, C. F. J. (1997), “A Method for Constructing Supersaturated Designs and its $E\left(s^{2}\right)$ -Optimality”, Canadian Journal of Statistics, Vol. 25, pp. 191-201.

[9] Wilson, R. M. (1972), "Cyclotomy and Difference Families is Elementary Abelian Groups”, Journal of Number Theory, Vol. 4, pp. 17-47.

[10] Wu, C. F. J. (1993), “Construction of Supersaturated Design Through Partially AliasedInteractions”, Biometrika,, Vol. 80, pp. 661-669. MR 1248029. Methpdol. 63 621-632 MR 1858407.

[11] Xu, H. and Wu, C. F. J. (2005), “Construction of Optimal Multi-level Supersaturated Designs”, The Annals of Statistics, Vol. 33, No. 6. Dec. 2005.

[12] Sun,Fasheng; Lin,K J Dennis; and Liu,Min-Qian(2011),”On the Construction of Optimal Mix-Level Supersaturated Designs”, Annals of Statistics,Vol.39, No.2,pp1310-1333. 